\title{
Research on Decision-Making Method of Bid Evaluation for Engineering Projects Based on Fuzzy DEA and Grey Relation
}

\author{
Yi Zhang* \\ School of Management, Hubei University of Technology, Wuhan430068, China
}

\begin{abstract}
At present, bid evaluation in China mainly adopts the method of traditional comprehensive scoring. And the method of analytic hierarchy process or fuzzy synthetic assessment is also in common use. However, those methods have their own weaknesses, e.g., having a strong subjectivity, lacking in analysis of quantitative indicators, and being unable to reflect the defects of the bidders and reasons for ineffectiveness, etc. This paper integrated fuzzy theory, data envelopment analysis (DEA) and grey relational analysis and proposed a systematic decision-making method of bid evaluation based on fuzzy DEA and grey relation for choosing proper bidders. For qualitative indicators, the method took matrix of optimal objective function values as matrix of qualitative indicator values by constructing $\mathrm{C}^{2} \mathrm{R}$ model on basis of membership matrix; for quantitative indicators, the method took matrix of correlative coefficients as matrix of quantitative indicator values by using the grey system theory. At last, this paper introduced a case study to confirm the rationality and feasibility of the decision-making method of bid evaluation, being valuable and helpful for assessment applications in other areas.
\end{abstract}

Keywords: Bid evaluation, engineering projects, fuzzy DEA, grey relation.

\section{INTRODUCTION}

As the development of China's construction project bidding system, a growing number of projects tend to determine the contractor through bidding. Therefore bid evaluation methods become a main concern. Bid evaluation is the core of the bidding, and the premise and indispensable step of scientific decision, which is an essential part of bidding. To some degree, scientificity and reasonability of the assessment method is of great significance in bidding and directly related to the success or failure of the entire bidding activity. Following the principles of scientificity, rationality, fairness, openness and impartiality, on the premise of satisfying the substantive requirements of the tender documents as well as possible, bid evaluation should conduct a full assessment of all aspects of the bidders, including quotation, schedule, quality, consumption of main material, plan of construction technology, performance, reputation, and preferential terms, etc. Instead of merely following the principle of the lowest price, bidding is a multi-attribute decision-making activity in essence.

Since bidding started quite late in China, its actual operation still faces many problems, especially in the aspects of bid evaluation method lacking research in theory and lacking experience in practice. Therefore, it is of great theoretic and realistic significance to study bid evaluation methods for engineering projects and establish a scientific, practical and feasible assessment model. At present, bid evaluation in China mainly adopts the method of traditional comprehensive scoring $[1,2]$. However this method does not have a rigorous mathematical reasoning, and relying on the subjective estimation of the experts, it is difficult for this method to avoid errors caused by artificial factors, which is against impartiality. The method of analytic hierarchy process [3-7] and fuzzy synthetic assessment [8] are also applied in bid evaluation [9-12]. But there are also weaknesses in these methods, e.g., having strong subjectivity, having illogical analysis of quantitative indicators, and failing to reflect the defects of the bidders and reasons for ineffectiveness, etc. In order to resolve these issues mentioned above, this paper integrated fuzzy theory, data envelopment analysis and grey relational analysis and proposed a systematic decision-making method of bid evaluation based on fuzzy DEA and grey relation for choosing proper bidders. For qualitative indicators, the method took matrix of optimal objective function values as matrix of qualitative indicator values by constructing $C^{2} R$ model on basis of membership matrix; for quantitative indicators, the method took matrix of correlative coefficients as matrix of quantitative indicator values by using the grey system theory. Finally, this paper introduced a case study to confirm the rationality and feasibility of the decision-making method of bid evaluation, being valuable and helpful for assessment applications in other areas.

The outline of the paper is as follows: The first part is to analyze and summarize the background and significance of the study; the second part is to establish a decision-making model of bid evaluation according to fuzzy DEA and grey relation; the third part shows the feasibility and rationality of the decision-making model of bid evaluation based on example analysis; the fourth part is the conclusion. 
2. DECISION-MAKING MODEL OF BID EVALUATION BASED ON FUZZY DEA AND GREY RELATION

\subsection{Establishing Indicators System of Bid Evaluation}

Selecting the assessment indicators is a very significant work. In the practical synthetic assessment activities, it is common to apply methods of Delphi, minimum mean square error, mini-max dispersion and correlative coefficient to choose indicators [13]. In this paper, Delphi method is applied to get the idea that the indicators system of bid evaluation is composed of five parts: Project Quotation, Project Duration, Project Quality, Construction Technology and Corporate Reputation.

\subsection{Determining the Matrix of Qualitative Indicator Values}

As for qualitative indicators like Project Quality, Construction Technology and Corporate Reputation, they are difficult to be described using appropriate mathematical language since they belong to the fuzzy variables and have the feature of unclear boundaries. Therefore, fuzzy theory should be employed in this study [14-18]. However, in actual applications, the method of fuzzy synthetic assessment merely reflect virtues or defect degree of various bidders, failing to reflect the defects of the bidders and reasons for ineffectiveness. On account of the reasons mentioned above, this paper tried to combine the method of fuzzy synthetic assessment with data envelopment analysis for such qualitative indicators. The specific procedures are shown as follows.

\subsubsection{Determining Qualitative Indicators Set and Assess- ment Grades Set}

It is assumed that $A=\left\{A_{1}, A_{2}, \cdots, A_{i}, \cdots, A_{m}\right\}$ $(i=1,2, \cdots, m)$ is the appraisal objects set and $m$ is the number of objects for assessment.

It is assumed that $B=\left\{B_{1}, B_{2}, \cdots, B_{j}, \cdots, B_{g}\right.$, $\left.B_{g+1}, B_{g+2}, \cdots, B_{n}\right\} \quad(j=1,2, \cdots, g, g+1, g+2, \cdots, n)$ is the assessment indicators set and $n$ is the number of assessment indicators. Among them, $B^{1}=\left\{B_{1}, B_{2}, \cdots, B_{g}\right\} \quad(j=1,2, \cdots, g)$ is the qualitative indicators set and $g$ is the number of qualitative indicators; $B^{2}=\left\{B_{g+1}, B_{g+2}, \cdots, B_{n}\right\}(j=g+1, g+2, \cdots, n)$ is the quantitative indicators set and $n-g$ is the number of quantitative indicators.

\section{It is assumed that $C=\left\{C_{1}, C_{2}, \cdots, C_{h}, \cdots, C_{d}\right\}$} $(h=1,2, \cdots, d)$ is the assessment grades set. It expresses the condition of each qualitative indicator. $d$ is the number of assessment grades and commonly divided into three to five levels, e.g., very poor, poor, medium, good, excellent.

\subsubsection{Building Membership Matrixes of Qualitative Indi- cators}

Aiming at each qualitative indicator $B_{j}(j=1,2, \cdots, g)$, we build a fuzzy relation matrix $R_{j}(j=1,2, \cdots, g)$, which is the membership matrix of the qualitative indicator $B_{j}$. As for the appraisal object $A_{i}(i=1,2, \cdots, m)$, It is assumed that the membership of the qualitative indicator $B_{j}$ can be assessed as the grade $C_{h}(h=1,2, \cdots, d)$ is $r_{i j h}$. Accordingly, we can obtain the assessment vector $r_{i j}$ of the simple object $A_{i}$ : $r_{i j}=\left(r_{i j 1}, r_{i j 2}, \cdots, r_{i j d}\right) \quad(i=1,2, \cdots, m)$, therefore $m$-objects assessment vectors form a overall membership matrix $R_{j}$ shown as follows:

$R_{j}=\left[\begin{array}{c}r_{1 j} \\ r_{2 j} \\ \vdots \\ r_{m j}\end{array}\right]=\left[\begin{array}{cccc}r_{1 j 1} & r_{1 j 2} & \cdots & r_{1 j d} \\ r_{2 j 1} & r_{2 j 2} & \cdots & r_{2 j d} \\ \vdots & \vdots & \ddots & \vdots \\ r_{m j 1} & r_{m j 2} & \cdots & r_{m j d}\end{array}\right](j=1,2, \cdots, g)$

\subsubsection{Constructing Fuzzy DEA Model}

On basis of the concept of "relative efficiency", DEA is a systematic method of assessing relatively effectiveness of decision-making units of the same type in accordance with the multi-indicators input and multi-indicators output [1925]. It is not only able to assess the relative effectiveness of each appraisal object, but also able to indicate the defects of each appraisal object and reasons for ineffectiveness. This paper will establish the most extensively used model $C^{2} R$ based on the membership matrix of the qualitative indicators.

Aiming at each qualitative indicator $B_{j}(j=1,2, \cdots, g)$, every appraisal object $\left\{A_{i}\right\}(i=1,2, \cdots, m)$ is selected as a decision-making unit of DEA model, with each grade $\left\{C_{h}\right\}$ $(h=1,2, \cdots, d)$ being the input or output of DEA model, and the transpose matrix $R_{j}^{T} \quad(j=1,2, \cdots, g)$ of the membership matrix $R_{j}(j=1,2, \cdots, g)$ of the qualitative indicator $B_{j}$ being input data and output data of decision-making units [26]. Aiming at each decision-making unit $A_{i}$, we suppose it has $p$-type input and $q$-type output, and the corresponding input vector is $X_{i}=\left(x_{1 i}, x_{2 i}, \cdots, x_{s i}, \cdots, x_{p i}\right)^{T}$ $(s=1,2, \cdots, p)$, and the corresponding output vector is $Y_{i}=\left(y_{1 i}, y_{2 i}, \cdots, y_{t i}, \cdots, y_{q i}\right)^{T} \quad(t=1,2, \cdots, q)$. Therefore $p+q=d$, with $d$ being the number of the assessment grades. At the same time, we present the input weights vector $V=\left(v_{1}, v_{2}, \cdots, v_{s}, \cdots, v_{p}\right)^{T}$ and the output weights vector $U=\left(u_{1}, u_{2}, \cdots, u_{t}, \cdots, u_{q}\right)^{T}$. 
As for the qualitative indicator $B_{1}$, the efficiency of decision-making unit $A_{1}$ will be assessed. The optimized model will be established, with the efficiency indicator $h_{11}$ of the decision-making unit $A_{1}$ being the objective function and the efficiency indicators of all the decision-making units (including the decision-making unit $A_{1}$ ) being constraints. The initial $C^{2} R$ model is a fractional mathematical plan. By applying the changes of Charnes-Cooper [27], a linear programming model can be got as follows:

$$
\begin{aligned}
& \max h_{11}=\sum_{t=1}^{q} u_{t} y_{t 1} \\
& \text { s.t. } \sum_{s=1}^{p} v_{s} x_{s i}-\sum_{t=1}^{q} u_{t} y_{t i} \geq 0 \quad i=1,2, \cdots, m \\
& \sum_{s=1}^{p} v_{s} x_{s 1}=1 \\
& v=\left(v_{1}, v_{2}, \cdots, v_{s}, \cdots, v_{p}\right)^{T} \geq 0 \\
& u=\left(u_{1}, u_{2}, \cdots, u_{t}, \cdots, u_{q}\right)^{T} \geq 0
\end{aligned}
$$

By resolving the linear programming model mentioned above, we can get the optimal objective function $h_{11}$ of the decision-making unit $A_{1}$ as for the qualitative indicator $B_{1}$. It is the manifestation of the decision-making unit $A_{1}$ in the aspect of the qualitative indicator $B_{1}$ and a decimal between 0 and 1 . Accordingly, we can obtain the manifestation vector of every decision-making unit in the aspect of the qualitative indicator $B_{1}: H_{1}=\left(h_{11}, h_{21}, \cdots, h_{m 1}\right)^{T}$, so that we can obtain the manifestation matrix $H$ of every decision-making unit $\left\{A_{i}\right\} \quad(i=1,2, \cdots, m) \quad$ on each qualitative indicator $B_{j}(j=1,2, \cdots, g)$, which is regarded as the matrix of qualitative indicator values (also the matrix of optimal objective function values) shown as follows:

$$
H=\left(H_{1}, \mathrm{H}_{2}, \ldots, \mathrm{H}_{g}\right)=\left(h_{i j}\right)_{m \times g}=\left[\begin{array}{cccc}
h_{11} & h_{12} & \cdots & h_{1 g} \\
h_{21} & h_{22} & \cdots & h_{2 g} \\
\vdots & \vdots & \ddots & \vdots \\
h_{m 1} & h_{m 2} & \cdots & h_{m g}
\end{array}\right]
$$

\subsection{Determining the Matrix of Quantitative Indicator Values}

The values of quantitative indicators like Project Quotation and Project Duration can be defined in accordance with the initial indicator values. This paper tried to use the grey system theory [28], taking the matrix of correlation coefficients as the matrix of quantitative indicator values.

It is assumed that the initial indicator values matrix $Z$ of the $n-g$ quantitative indicators $B_{j}(j=g+1, g+2, \cdots, n)$ of $m$-objects $A_{i} \quad(i=1,2, \cdots, m)$ is shown as follows:

$$
Z=\left(z_{i j}\right)_{m \times(n-g)}=\left[\begin{array}{c}
Z_{1} \\
Z_{2} \\
\vdots \\
Z_{m}
\end{array}\right]=\left[\begin{array}{cccc}
z_{1, g+1} & z_{1, g+2} & \cdots & z_{1 n} \\
z_{2, g+1} & z_{2, g+2} & \cdots & z_{2 n} \\
\vdots & \vdots & \ddots & \vdots \\
z_{m, g+1} & z_{m, g+2} & \cdots & z_{m n}
\end{array}\right]
$$

\subsubsection{Determining the Optimal Indicator Values Set}

It is assumed that the optimal indicator values set is $Z_{0}=\left(z_{0, g+1}, z_{0, g+2}, \cdots, z_{0 n}\right)$ and $z_{0 j}(j=g+1, g+2, \cdots, n)$ is the optimal value of the quantitative indicator $B_{j}(j=g+1, g+2, \cdots, n)$.

When the assessment indicator is cost, $z_{0 j}=\min \left(z_{1 j}, z_{2 j}, \cdots, z_{m j}\right)$; When the assessment indicator is effective, $z_{0 j} \stackrel{\max }{=}\left(z_{1 j}, z_{2 j}, \cdots, z_{m j}\right)$. The matrix $D$ can be constructed after selecting the optimal indicator values set as follows:

$$
D=\left[\begin{array}{c}
Z_{0} \\
Z_{1} \\
Z_{2} \\
\vdots \\
Z_{m}
\end{array}\right]=\left[\begin{array}{cccc}
z_{0, g+1} & z_{0, g+2} & \cdots & z_{0 n} \\
z_{1, g+1} & z_{1, g+2} & \cdots & z_{1 n} \\
z_{2, g+1} & x_{2, g+2} & \cdots & x_{2 n} \\
\vdots & \vdots & & \vdots \\
x_{m, g+1} & x_{m, g+2} & \cdots & x_{m n}
\end{array}\right]
$$

\subsubsection{Standardizing Assessment Indicator Values}

Assessment indicators often have different dimensions and magnitude, so they are not directly comparable. In order to ensure the reliability of the results, we need normalize the matrix $D$. Methods of normalization processing, efficacy coefficient and extremum processing are generally used in this process. This paper adopts the extremum processing method.

It is assumed that $M_{j}=\max \left(z_{0 j}, z_{1 j}, z_{2 j}, \cdots, z_{m j}\right)$; $m_{j}=\min \left(z_{0 j}, z_{1 j}, z_{2 j}, \cdots, z_{m j}\right)$

For cost indicators,

$z_{i j}^{*}=\frac{M_{j}-z_{i j}}{M_{j}-m_{j}} \quad(j=g+1, g+2, \cdots, n)$

For effective indicators,

$z_{i j}^{*}=\frac{z_{i j}-m_{j}}{M_{j}-m_{j}} \quad(j=g+1, g+2, \cdots, n)$

Standardization matrix $F$ can be got as follows:

$F=\left[\begin{array}{c}Z_{0}^{*} \\ Z_{1}^{*} \\ Z_{2}^{*} \\ \vdots \\ Z_{m}^{*}\end{array}\right]=\left[\begin{array}{cccc}z_{0, g+1}^{*} & z_{0, g+2}^{*} & \cdots & z_{0 n}^{*} \\ z_{1, g+1}^{*} & z_{1, g+2}^{*} & \cdots & z_{1 n}^{*} \\ z_{2, g+1}^{*} & z_{2, g+2}^{*} & \cdots & z_{2 n}^{*} \\ \vdots & \vdots & & \vdots \\ z_{m, g+1}^{*} & z_{m, g+2}^{*} & \cdots & z_{m n}^{*}\end{array}\right]$ 


\subsubsection{Determining the Matrix of Correlation Coefficients}

According to the grey relation method, the correlation coefficient of the quantitative indicator $B_{j}(j=g+1, g+2, \cdots, n)$ of the appraisal object $A_{i}(i=1,2, \cdots, m)$ with the optimal indicator $B_{0 j}$ can be obtained as follows:

$e_{i j}=\frac{\min _{i} \min _{j}\left|z_{i j}^{*}-z_{i j}\right|+\rho \max _{i} \max _{j}\left|z_{i j}^{*}-z_{i j}\right|}{\left|z_{i j}^{*}-z_{i j}\right|+\rho \max _{i} \max _{j}\left|z_{i j}^{*}-z_{i j}\right|}$

$\rho$ is the distinguish coefficient and generally taken $\rho=0.5$. It is clear that the correlation coefficient matrix $E$ which is regarded as the matrix of quantitative indicator values can be attained as follows:

$$
E=\left(e_{i j}\right)_{m \times(n-g)}=\left[\begin{array}{cccc}
e_{1, g+1} & e_{1, g+2} & \cdots & e_{1 n} \\
e_{2, g+1} & e_{2, g+2} & \cdots & e_{2 n} \\
\vdots & \vdots & \ddots & \vdots \\
e_{m, g+1} & e_{m, g+2} & \cdots & e_{m n}
\end{array}\right]
$$

\subsection{Determining the Matrix of Assessment Indicator Values}

The matrix $G$ of assessment indicator values can be constructed on basis of the matrix $H$ of qualitative indicator values and the matrix $E$ of quantitative indicator values shown as follows:

$$
G=[H, E]=\left[\begin{array}{cccccccc}
h_{11} & h_{12} & \cdots & h_{1 g} & e_{1, g+1} & e_{1, g+2} & \cdots & e_{1 n} \\
h_{21} & h_{22} & \cdots & h_{2 g} & e_{2, g+1} & e_{2, g+2} & \cdots & e_{2 n} \\
\vdots & \vdots & \ddots & \vdots & \vdots & \vdots & \ddots & \vdots \\
h_{m 1} & h_{m 2} & \cdots & h_{m g} & e_{m, g+1} & e_{m, g+2} & \cdots & e_{m n}
\end{array}\right]
$$

\subsection{Determining Assessment Indicator Weight Vector}

The determination of assessment indicator weight always uses methods of Delphi, AHP, $\mathrm{G}_{1}$ and $\mathrm{G}_{2}$. This paper adopts the method of Delphi.

Assume $W=\left\{w_{1}, w_{2}, \cdots, w_{g}, w_{g+1}, w_{g+2}, \cdots, w_{n}\right\}^{T}$ as the assessment indicator weights vector.

\subsection{Determining Comprehensive Assessment Results}

The matrix $K$ can be determined according to the matrix $G$ of assessment indicator values and the assessment indicator weights vector $W$ as follows:

$$
\begin{aligned}
& K=G \times W \\
& =\left[\begin{array}{cccccccc}
h_{11} & h_{12} & \cdots & h_{1 g} & e_{1, g+1} & e_{1, g+2} & \cdots & e_{1 n} \\
h_{21} & h_{22} & \cdots & h_{2 g} & e_{2, g+1} & e_{2, g+2} & \cdots & e_{2 n} \\
\vdots & \vdots & \ddots & \vdots & \vdots & \vdots & \ddots & \vdots \\
h_{m 1} & h_{m 2} & \cdots & h_{m g} & e_{m, g+1} & e_{m, g+2} & \cdots & e_{m n}
\end{array}\right] \times\left[\begin{array}{c}
w_{1} \\
w_{2} \\
\vdots \\
w_{n}
\end{array}\right]=\left[\begin{array}{c}
\delta_{1} \\
\delta_{2} \\
\vdots \\
\delta_{m}
\end{array}\right](12)
\end{aligned}
$$

The maximum value of $\delta_{i}(i=1,2, \cdots, m)$ will be taken as the successful bidder.

\section{CASE STUDY}

\subsection{Case Profile}

We suppose that there is a project bidding. After the verification of the qualification, four bidders $A=\left\{A_{1}, A_{2}, A_{3}, A_{4}\right\}$ enter the last bid evaluation phase. By Delphi method, we determine that the assessment indicators set is consisted of the five indicators, namely $B=$ \{Project Quality ( $\left.B_{1}\right)$, Construction Technology $\left(B_{2}\right)$, Corporate Reputation ( $B_{3}$ ), Project Quotation ( $B_{4}$ ), Project Duration $\left.\left(B_{5}\right)\right\}$, among which the quantitative indicators set is $B^{1}=\left\{B_{1}, B_{2}, B_{3}\right\}$ and the qualitative indicators set is $B^{2}=\left\{B_{4}, B_{5},\right\}$. The initial indicator values for quantitative indicators are displayed in Table $\mathbf{1}$ below:

Table 1. Original indicator values of quantitative indicator.

\begin{tabular}{|c|c|c|}
\hline Bidders & $\begin{array}{c}\text { Project Quotation (B }) \\
\text { (million-yuan) }\end{array}$ & $\begin{array}{c}\text { Project Duration (B }) \\
\text { (Days) }\end{array}$ \\
\hline \hline $\mathrm{A}_{1}$ & 25 & 545 \\
\hline $\mathrm{A}_{2}$ & 24 & 480 \\
\hline $\mathrm{A}_{3}$ & 23.5 & 500 \\
\hline $\mathrm{A}_{4}$ & 24.8 & 520 \\
\hline
\end{tabular}

\subsection{Determining the Matrix of Qualitative Indicator Values}

\subsubsection{Determining Qualitative Indicators Set and Assess- ment Grades Set}

It is easy to ascertain that the qualitative indicators set $B^{1}=\left\{\right.$ Project Quality $\left(B_{1}\right)$, Construction Technology $\left(B_{2}\right)$, Corporate Reputation $\left.\left(B_{3}\right)\right\}$, while assessment levels set $C=\left\{\right.$ very poor $\left(C_{1}\right)$, poor $\left(C_{2}\right)$, medium $\left(C_{3}\right)$, good $\left(C_{4}\right)$, excellent $\left.\left(C_{5}\right)\right\}$ can also be determined. We invited nine experts of bid evaluation to make a fuzzy assessment of 4 bidders' manifestation on each qualitative indicator. The results are listed in Tables 2-4.

\subsubsection{Building the Membership Matrix of Qualitative Indi- cator}

The membership matrix $R_{j}(j=1,2,3)$ can be constructed aiming at each qualitative indicator $B_{j} \quad(j=1,2,3)$ according to the equation (1) on basis of the Table $2 \sim 4$ as follows: 
Table 2. Assessment valuation results of quality $\left(B_{1}\right)$.

\begin{tabular}{|c|c|c|c|c|c|}
\hline Quality (B $\mathbf{B}_{\mathbf{1}}$ & $\mathbf{C}_{\mathbf{1}}$ & $\mathbf{C}_{\mathbf{2}}$ & $\mathbf{C}_{\mathbf{3}}$ & $\mathbf{C}_{\mathbf{4}}$ & $\mathbf{C}_{\mathbf{5}}$ \\
\hline \hline $\mathrm{A}_{1}$ & 0 & 1 & 2 & 3 & 1 \\
\hline $\mathrm{A}_{2}$ & 1 & 3 & 4 & 1 & 0 \\
\hline $\mathrm{A}_{3}$ & 2 & 3 & 3 & 2 & 1 \\
\hline $\mathrm{A}_{4}$ & 0 & 3 & 3 & 3 \\
\hline
\end{tabular}

Table 3. Assessment valuation results of technology $\left(B_{2}\right)$.

\begin{tabular}{|c|c|c|c|c|c|}
\hline Technology $\left(\mathbf{B}_{2}\right)$ & $\mathbf{C}_{\mathbf{1}}$ & $\mathbf{C}_{\mathbf{2}}$ & $\mathbf{C}_{\mathbf{3}}$ & $\mathbf{C}_{\mathbf{4}}$ & $\mathbf{C}_{\mathbf{5}}$ \\
\hline \hline $\mathrm{A}_{1}$ & 1 & 4 & 3 & 1 & 3 \\
\hline $\mathrm{A}_{2}$ & 0 & 2 & 2 & 1 & 0 \\
\hline $\mathrm{A}_{3}$ & 3 & 3 & 4 & 2 & 1 \\
\hline $\mathrm{A}_{4}$ & 0 & 2 & 4 & 2 \\
\hline
\end{tabular}

Table 4. Assessment valuation results of reputation $\left(B_{3}\right)$.

\begin{tabular}{|c|c|c|c|c|c|}
\hline Reputation $\left(\mathbf{B}_{3}\right)$ & $\mathbf{C}_{\mathbf{1}}$ & $\mathbf{C}_{\mathbf{2}}$ & $\mathbf{C}_{\mathbf{3}}$ & $\mathbf{C}_{\mathbf{4}}$ & $\mathbf{C}_{\mathbf{5}}$ \\
\hline \hline $\mathrm{A}_{1}$ & 2 & 4 & 2 & 1 & 0 \\
\hline $\mathrm{A}_{2}$ & 0 & 4 & 3 & 2 & 1 \\
\hline $\mathrm{A}_{3}$ & 0 & 2 & 5 & 1 & 0 \\
\hline $\mathrm{A}_{4}$ & 1 & 2 & 5 & 2 \\
\hline
\end{tabular}

$$
\begin{aligned}
& R_{1}=\left[\begin{array}{ccccc}
0 & 0.111 & 0.223 & 0.333 & 0.333 \\
0.111 & 0.333 & 0.445 & 0.111 & 0 \\
0.223 & 0.333 & 0.333 & 0.111 & 0 \\
0 & 0.333 & 0.333 & 0.223 & 0.111
\end{array}\right] \\
& R_{2}=\left[\begin{array}{ccccc}
0.111 & 0.445 & 0.333 & 0.111 & 0 \\
0 & 0.222 & 0.222 & 0.334 & 0.222 \\
0.333 & 0.333 & 0.223 & 0.111 & 0 \\
0 & 0.222 & 0.445 & 0.222 & 0.111
\end{array}\right] \\
& R_{3}=\left[\begin{array}{ccccc}
0.222 & 0.445 & 0.222 & 0.111 & 0 \\
0 & 0.445 & 0.333 & 0.111 & 0.111 \\
0 & 0.222 & 0.334 & 0.222 & 0.222 \\
0.111 & 0.222 & 0.556 & 0.111 & 0
\end{array}\right]
\end{aligned}
$$

\subsubsection{Constructing Fuzzy DEA Model}

We choose very poor $\left(C_{1}\right)$, poor $\left(C_{2}\right)$, medium $\left(C_{3}\right)$ as input and good $\left(C_{4}\right)$ and excellent $\left(C_{5}\right)$ as output of the DEA model. Meanwhile, we take all bidders $A_{i}(i=1,2,3,4)$ as the decision-making units of DEA model, and present the input weights vector $V=\left(v_{1}, v_{2}, v_{3}\right)^{T}$ and the output weights vector $U=\left(u_{1}, u_{2}\right)^{T}$.

As for qualitative indicator "Project Quality $\left(B_{1}\right)$ ", we use the transpose matrix $R_{1}^{T}$ of its membership matrix $R_{1}$ as input data and output data of decision-making unit. Now efficiency of the bidder $A_{1}$ will be assessed. According to the model (2), we can construct the following linear programming model:

$$
\begin{aligned}
& \max h_{11}=0.333 u_{1}+0.333 u_{2} \\
& \text { s.t. } 0.111 v_{2}+0.223 v_{3}-0.333 u_{1}-0.333 u_{2} \geq 0 \\
& 0.111 v_{1}+0.333 v_{2}+0.445 v_{3}-0.111 u_{1} \geq 0 \\
& 0.223 v_{1}+0.333 v_{2}+0.333 v_{3}-0.111 u_{1} \geq 0 \\
& 0.333 v_{2}+0.333 v_{3}-0.223 u_{1}-0.111 u_{2} \geq 0 \\
& 0.111 v_{2}+0.223 v_{3}=1 \\
& V=\left(v_{1}, v_{2}, v_{3}\right)^{T} \geq 0 \\
& U=\left(u_{1}, u_{2}\right)^{T} \geq 0
\end{aligned}
$$


Using LINDO software, we can get $h_{11}=1.0000$, and this is the manifestation of the project bidders $A_{1}$ in the aspect of project quality $B_{1}$, which is also the optimal objective function value. Accordingly, we can get the manifestation of the other three bidders in the aspect of project quality $B_{1}: h_{21}=0.1670, h_{31}=0.2232, h_{41}=0.4485$. Thus we can know the manifestation of all decision-making units in the aspect of project quality $\left(B_{1}\right)$ :

$$
H_{1}=\left(h_{11}, h_{21}, h_{31}, h_{41}\right)^{T}=(1.0000,0.1670,0.2232,0.4485)^{T} .
$$

Similarly, as for the construction technology $\left(B_{2}\right)$, all decision-making units' manifestation on it can be shown in:

$$
H_{2}=\left(h_{12}, h_{22}, h_{32}, h_{42}\right)^{T}=(0.2216,1.0000,0.3308,0.6647)^{T}
$$

As for corporate reputation ( $\left.B_{3}\right)$, all decision-making units' manifestation on it can be shown in:

$$
H_{3}=\left(h_{13}, h_{23}, h_{33}, h_{43}\right)^{T}=(0.7500,0.5000,1.0000,0.5023)^{T}
$$

Therefore the manifestation matrix $H$ of every decisionmaking unit $A_{i}(i=1,2,3,4)$ on each qualitative indicator $B_{j}(j=1,2,3)$ can be displayed according to the equation (3), which is also the matrix of optimal objective function value shown as follows:

$$
H=\left(H_{1}, H_{2}, H_{3}\right)=\left(h_{i j}\right)_{4 \times 3}=\left[\begin{array}{lll}
1.0000 & 0.2216 & 0.7500 \\
0.1670 & 1.0000 & 0.5000 \\
0.2232 & 0.3308 & 1.0000 \\
0.4485 & 0.6647 & 0.5023
\end{array}\right]
$$

\subsection{Determining the Matrix of quantitative Indicator} Values

The initial indicator values matrix $Z$ of the quantitative indicators $B_{4}$ and $B_{5}$ can be got according to the equation (4) on basis of the table 1 shown as follows:

$$
Z=\left(z_{i j}\right)_{4 \times 2}=\left[\begin{array}{c}
Z_{1} \\
Z_{2} \\
Z_{3} \\
Z_{4}
\end{array}\right]=\left[\begin{array}{cc}
2500 & 545 \\
2400 & 480 \\
2350 & 500 \\
2480 & 520
\end{array}\right]
$$

\subsubsection{Determining the Optimal Indicator Values Set}

The quantitative indicators $B_{4}$ and $B_{5}$ are cost indicators, so the optimal indicator values set is $Z_{0}=(2350,480)$. The matrix $D$ can be constructed according to the equation (5) as follows:

$$
D=\left[\begin{array}{l}
Z_{0} \\
Z_{1} \\
Z_{2} \\
Z_{3} \\
Z_{4}
\end{array}\right]=\left[\begin{array}{ll}
2350 & 480 \\
2500 & 545 \\
2400 & 480 \\
2350 & 500 \\
2480 & 520
\end{array}\right]
$$

\subsubsection{Standardizing Assessment Indicator Values}

The quantitative indicators $B_{4}$ and $B_{5}$ are cost indicators, so the Standardization matrix $F$ can be constructed according to the equation (6) and (8) as follows:

$$
F=\left[\begin{array}{c}
Z_{0}^{*} \\
Z_{1}^{*} \\
Z_{2}^{*} \\
Z_{3}^{*} \\
Z_{4}^{*}
\end{array}\right]=\left[\begin{array}{cc}
1 & 1 \\
0 & 0 \\
0.6667 & 1 \\
1 & 0.6923 \\
0.1333 & 0.3846
\end{array}\right]
$$

\subsubsection{Determining the Matrix of Correlation Coefficients}

Firstly, it is easy to determine that

$$
\left|z_{i j}^{*}-z_{i j}\right|=\left[\begin{array}{cc}
0 & 0 \\
1 & 1 \\
0.3333 & 0 \\
0 & 0.3077 \\
0.8667 & 0.6154
\end{array}\right]
$$

Secondly, it is easy to determine that

$$
\min _{i} \min _{j}\left|z_{i j}^{*}-z_{i j}\right|=0, \quad \max _{i} \max _{j}\left|z_{i j}^{*}-z_{i j}\right|=1 \text { and } \rho \text { is }
$$

generally taken $\rho=0.5$, so $e_{i j}$ can be obtained according to the equation (9) as follows:

$$
\begin{aligned}
& e_{i j}=\frac{\min _{i} \min _{j}\left|z_{i j}^{*}-z_{i j}\right|+\rho \max _{i} \max _{j}\left|z_{i j}^{*}-z_{i j}\right|}{\left|z_{i j}^{*}-z_{i j}\right|+\rho \max _{i} \max _{j}\left|z_{i j}^{*}-z_{i j}\right|} \\
& =\frac{0.5}{\left|z_{i j}^{*}-z_{i j}\right|+0.5}
\end{aligned}
$$

Finally, the matrix $E$ of correlation coefficients which is regarded as the matrix of quantitative indicator values can be attained according to the equation (10) as follows:

$$
E=\left(e_{i j}\right)_{4 \times 2}=\left[\begin{array}{ll}
0.3333 & 0.3333 \\
0.6000 & 1.0000 \\
1.0000 & 0.6190 \\
0.3658 & 0.4483
\end{array}\right]
$$

\subsection{Determining the Matrix of Assessment Indicator Values}

The matrix $G$ of assessment indicator values can be constructed on basis of the matrix $H$ of qualitative indicator values and the matrix Eof quantitative indicator values according to the equation (11) as follows:

$$
G=[H, E]=\left[\begin{array}{lllll}
1.0000 & 0.2216 & 0.7500 & 0.3333 & 0.3333 \\
0.1670 & 1.0000 & 0.5000 & 0.6000 & 1.0000 \\
0.2232 & 0.3308 & 1.0000 & 1.0000 & 0.6190 \\
0.4485 & 0.6647 & 0.5023 & 0.3658 & 0.4483
\end{array}\right]
$$




\subsection{Determining Assessment Indicator Weight Vector}

The assessment indicator weight vector can be attained by adopting the method of Delphi as follows: $W=\left\{w_{1}, w_{2}, w_{3}, w_{4}, w_{5}\right\}^{T}=\{0.20,0.15,0.05,0.40,0.20\}^{T}$.

\subsection{Determining Comprehensive Assessment Results}

The comprehensive assessment vector $K$ can be attained based on assessment indicator values matrix $G$ and the assessment indicator weight vector $W$ according to the equation (12) as follows:

$$
K=G \times W=\left[\begin{array}{l}
\delta_{1} \\
\delta_{2} \\
\delta_{3} \\
\delta_{4}
\end{array}\right]
$$

$=\left[\begin{array}{lllll}1.0000 & 0.2216 & 0.7500 & 0.3333 & 0.3333 \\ 0.1670 & 1.0000 & 0.5000 & 0.6000 & 1.0000 \\ 0.2232 & 0.3308 & 1.0000 & 1.0000 & 0.6190 \\ 0.4485 & 0.6647 & 0.5023 & 0.3658 & 0.4483\end{array}\right] \times\left[\begin{array}{c}0.20 \\ 0.15 \\ 0.05 \\ 0.40 \\ 0.20\end{array}\right]$

$$
=\left[\begin{array}{l}
0.4704 \\
0.6484 \\
0.6681 \\
0.4505
\end{array}\right]
$$

It is easy to determine that " $\delta_{3}>\delta_{2}>\delta_{1}>\delta_{4}$ ", so we take the bidder $A_{3}$ as the successful bidder.

\section{CONCLUSION}

(1) Bid evaluation is the core of the bidding, and the premise and indispensable step of scientific decision. This paper integrated fuzzy theory, data envelopment analysis and grey relational analysis and proposed a systematic decisionmaking method of bid evaluation based on fuzzy DEA and grey relation for choosing proper bidders. This approach can not only quantify the qualitative indicators and analyze the qualitative indicators and the quantitative indicators, but also can assess the relative effectiveness of each appraisal object and find out their defects and reasons for ineffectiveness.

(2) The greatest feature is that the method doesn't have a strict requirement to the size of the sample and needn't typical regularities of distribution. The most prominent feature of this method is that it has no strict requirements for the amount of samples and does not need the typical distribution rule. Its calculation method is simple, and has rigorous logical reasoning and mathematical foundation. And at the same time it has some reference value for the assessment in other application domains.

(3) In actual bidding evaluation activities, we need consider a large number of assessment indicators. This paper only considered several typical function indicators, which can also be subdivided, so the assessment indicators system should be further perfected.

\section{CONFLICT OF INTEREST}

The author confirms that this article content has no conflict of interest.

\section{ACKNOWLEDGEMENTS}

This work is supported by the Youth Fund Program of Humanities and Social Science Research of Ministry of Education of China (Program name: Research on Index System and Decision-Making Method of Bid Evaluation for Engineering Projects Based on D-S Evidence Theory from the Perspective of Green; Grant No.14YJC630201).

\section{REFERENCES}

[1] L Chen, Y Z Wang, "Research on TOPSIS integrated evaluation and decision method based on entropy coefficient," Control and Decision, vol.18, pp.456-459, 2003.

[2] H L Zou, S S Zhao, L X Zhang, "Evaluation method for engineering tenders based on synthetic analysis of quantitative and qualitative indexes", Journal of the China Railway Society, vol. 22, no. 6, pp. $112-115,2000$.

[3] T L Satty, "Modeling unstructured decision problems: a theory of analytic hierarchies", In: Proceedings of the $1^{\text {st }}$ International Conference on Mathematical Modeling, 1977, pp. 59-77.

[4] T L Satty, "A scaling method for priorities in hierarchical structures", Journal of Mathematical Psychology, vol. 1, no. 1, pp. 5768, 1978.

[5] T L Satty, Eds., The analytic hierarchy process, New York: McGraw-Hill: New York, 1980.

[6] T L Satty, "Axiomatic foundation of the analytic hierarchy process", Management Science, vol. 23, no. 7, pp. 851-855, 1986.

[7] T L Satty, L G Vargas, "Uncertainty and rank order in the analytic hierarchy process", European Journal of Operational Research, vol. 32, no. 1, pp. 107-117, 1987.

[8] J C Wang, "Multi-indexes fuzzy comprehensive judgment", Journal of Kunming University of Technology, vol.23, no.4, pp. 69-71, 1998.

[9] L T Shen, Q M Li, "Application of analytic hierarchy process in construction engineering bid evaluation", Construction Technology, vol. 34, pp. 64-66, 2005.

[10] B Pan, D R Zhang, "Evaluation for government purchasing bidding projects: A comprehensive analysis on the basis of vague mathematics", Systems Engineering, vol. 25, pp. 97-100, 2007.

[11] X P Zang, P Li, J Zhang, S D Ding, "Application of improved fuzzy integrating assessment in Evaluation", Journal of Jiangsu University of Science and Technology (Natural Science Edition), vol. 21, no. 6, pp. 9-12, 2007.

[12] Q Huang, W H Qiu, “A new method VE-AHP and It's application in bid assessment of the lift project," Chinese Journal of Management Science, vol.11, pp.164-167, 2003.

[13] Y J Guo, The Theory, Method and Application of Comprehensive Evaluation, Science Press: Beijing, 2007.

[14] L. A. Zadeh, "Fuzzy sets," Information and Control, vol. 8, pp. 338-353, 1965.

[15] R Bellman, L A Zadeh, "Decision making in a fuzzy environment" Management Science, vol. 17, no.4, pp. 141-164, 1970.

[16] S M Baas, H Kwakernaak, "Rating and ranking of multiple aspect alternative using fuzzy sets" Automatica, vol. 13, no.1, pp. 47-58, 1977.

[17] H J Zimmermann, Fuzzy Set Theory and Its Applications, Boston, Massachusetts: Kluwer Academic Publishers, 1977.

[18] H J Zimmermann, Fuzzy Set, Decision Making, and Expert System, Boston, Massachusetts: Kluwer, 1987. 
[19] L. M. Seiford, "Data envelopment analysis: The evolution of state of the art(1978-1995)," Journal of Production Analysis, vol. 7, pp. 99-137, 1996.

[20] A. Charnes, W. W. Cooper and E. Rhodes. "Measuring the efficiency of decision making units," European Journal of Operational Research, vol. 2, pp. 429-444, 1978.

[21] A Charnes, W W Cooper and B Golany, "Foundations of data envelopment analysis for pareto-koopmans efficient empirical production functions", Journal of Econometrics, vol. 30, pp. 91-107, 1985.

[22] G Yu, Q L Wei and P A Brockett, "Generalized data envelopment analysis model", Annals of Operations Research, pp. 47-89, 1996.

[23] Q L Wei, Date Envelopment Analysis. Science Press: Beijing, 2004.
[24] Y P Liu, Z G Lin and Z Y Shen, "Effective data envelopment analysis method for ranking decision making units," Systems Engineering Theory and Practice, vol. 3, pp. 112-116, 2006.

[25] P. Andersen and N. C. Petersen, "A procedure for ranking efficient units in data envelopment analysis," Management Science, vol. 39, pp.1261-1264, 1993.

[26] D Du, Q H Pang and Y Wu, Modern Comprehensive Evaluation Method and Case Selection. Tsinghua University Press: Beijing, 2008.

[27] A. Charnes, W. W. Cooper, "Programming with linear fractional functional," Naval Research Logistics Quarterly, vol. 9, pp.181$185,1962$.

[28] J L Deng, Grey Forecast and Decision, Huazhong Science and Technology University Press: Wuhan, 1998.

(C) Yi Zhang; Licensee Bentham Open.

This is an open access article licensed under the terms of the Creative Commons Attribution Non-Commercial License (http://creativecommons.org/licenses/by-nc/3.0/) which permits unrestricted, non-commercial use, distribution and reproduction in any medium, provided the work is properly cited. 